ISSN 0001-6002/2001/43/2/50-54

Acta Médica Costarricense,(C2001

Colegio de Médicos y Cirujanos

Revisiones

\title{
Importancia Clínica de la Proteinuria en Diabetes Mellitus
}

\author{
Valentín Figueroa, ${ }^{1}$ Karla M Urroz-Cárcamo, ${ }^{1}$ Carlos Arguedas-Chaverri ${ }^{1}$
}

Justificación: Ante la creciente incidencia de Diabetes Mellitus en la población joven, y en vista del pobre nivel de control metabólico alcanzado mundialmente para dicha enfermedad, es necesario encontrar métodos sencillos, prácticos y a nuestro alcance, para detectar en forma muy temprana la aparición de las complicaciones crónicas propias de la Diabetes. Así se lograría evitar, ó al menos enlentecer, la evolución natural de una enfermedad muy limitante para quien no conoce lo suficiente de cómo vivir con ella, o una vez advertido y debidamente instruido en sus implicaciones, hace caso omiso de lo que se recomienda.

Objetivo: Revisar la más reciente evidencia de utilidad y disponibilidad de los distintos métodos de evaluación de la albuminuria, para mejorar el abordaje del paciente diabético, con o sin nefropatía.

Metodología: Revisión bibliográfica de publicaciones de los últimos 10 años.

Conclusiones: 1) A todo paciente DM Tipo 1 luego de 5 años de ser diagnosticado y a todo paciente DM tipo 2 al momento del diagnóstico, debe realizársele medición de proteinuria. 2) Las tiras reactivas para determinar la micro y macroalbuminuria son confiables en relación con la medición de la orina de 24 horas. 3) Los factores más importantes dentro de la evolución de la nefropatía diabética y que exigen un agresivo manejo son: un buen control metabólico y el manejo antihipertensivo. 4) Los otros factores son importantes, pero su intervención es discutible.

Descriptores: Diabetes Mellitus, Nefropatía Diabética, Albuminuria, Insuficiencia Renal.

Recibido: 03 de julio de 2000

Aceptado: 24 de abril de 2001

Albuminuria, es definida como la excreción urinaria de albúmina (EUA) sobre el valor normal $(>30 \mathrm{mg} / 24 \mathrm{~h}){ }^{1}$

Dicho parámetro se ha constituido en el principal marcador para detectar la presencia de nefropatía diabética (ND) en forma incipiente, además de ser uno de los factores de riesgo para enfermedad cardiovascular. ${ }^{2-10}$

La Diabetes Mellitus (DM) es la principal causa de enfermedad renal terminal en Estados Unidos, Europa y Costa Rica (comunicación personal. Mata O, 1999), y esta conlleva a

\footnotetext{
Abreviaturas: ND, Nefropatía Diabética; DM, Diabetes Mellitus; HTA, Hipertensión Arterial; FG, Filtración Glomerular; EUA, Excreción urinaria de albúmina; DCCT, Diabetes Control and Complications Trial; UKPDS, United Kingdom Prospective Diabetes Study; HbA1c.

${ }^{1}$ Servicio de Medicina Interna, Hospital México.
}

Correspondencia: Karla María Urroz Cárcamo. Apdo. 1181-4050 Alajuela, Costa Rica. una alta morbilidad y mortalidad, con costos económicos que exceden los $\$ 2$ billones anualmente. ${ }^{11,12}$

La principal medida para su adecuado manejo es la prevención. La medición de la proteinuria constituye una valiosa ayuda para el adecuado abordaje y manejo del paciente diabético, con el fin de prevenir el progreso hacia la insuficiencia renal. La presencia de proteinuria refleja lesiones glomerulares, ya que es el sitio encargado de realizar el ultrafiltrado de plasma, aunque también puede tener un origen a nivel tubular. El aumento de la permeabilidad a macromoléculas como proteínas se acompaña de inflamación tubulo-intersticial que lleva a un deterioro progresivo de la función renal.

\section{Historia natural y epidemiología de la nefropatía diabética}

Para hablar de la proteinuria se requiere conocer la historia natural de la ND. Cerca del 20 al 30\% de los pacientes DM tipo 1 ó 2 desarrollan evidencia de ND. ${ }^{11}$. Estudios epidemiológicos en los indígenas Pima demuestran que la microalbuminuria se presentó en el $8 \%$ de aquellos con glucosa normal, 
en el $15 \%$ de aquellos con intolerancia a la glucosa y en el $47 \%$ de aquellos con DM. ${ }^{13}$

En los pacientes DM tipo 1, la microalbuminuria típicamente se detecta entre los 5 y 10 años de inicio de la enfermedad. ${ }^{12}$ Sin ninguna intervención específica, el $80 \%$ de ellos tiene una tasa de incremento de la EUA del 10 al $20 \%$ por año, y progresaron hacia una nefropatía abierta (albuminuria $>$ de $300 \mathrm{mg} / 24 \mathrm{~h}$ ) en un período de 10 a 15 años.

Sin intervención terapéutica, los pacientes con nefropatía abierta comienzan a disminuir su filtración glomerular (FG) con una variabilidad individual de $2-20 \mathrm{ml} / \mathrm{min} /$ año.

La enfermedad renal terminal (creatinina $>1,5 \mathrm{mg} / \mathrm{dl}$ y aclaramiento de creatinina $<$ del $80 \%$ del predicho o $<$ de $10 \mathrm{ml} /$ min) se desarrolla en el 50\% de los diabéticos con nefropatía abierta en un período de 10 años y en el $75 \%$ a los 20 años después de efectuado el diagnóstico de DM. ${ }^{11}$

En relación con los DM tipo 2, una alta proporción (50\%) puede tener microalbuminuria o aún nefropatía abierta, luego de corto tiempo del diagnóstico, ya que el inicio de la DM 2 es insidioso. Además, pueden tener hipertensión arterial asociada al momento de la presentación de la DM. ${ }^{11,12}$ Sin intervención especifica, del 20 al $40 \%$ de los pacientes con microalbuminuria progresan hacia nefropatía abierta; 20 años después del inicio de ésta, solo el $20 \%$ progresa hacia enfermedad renal terminal. ${ }^{11}$ La caída de la FG tiene variabilidad individual, pero puede no diferir sustancialmente de lo visto en DM tipo 1.

\section{Investigación de la proteinuria}

En los pacientes DM tipo 1 la proteinuria se debe investigar, idealmente, luego de la pubertad o después de 5 años de iniciada la enfermedad. En los DM tipo 2 se les debe investigar ésta desde el momento del diagnóstico, por las razones antes expuestas. ${ }^{11}$

La investigación se puede realizar por medio de dos métodos confiables que dan resultados comparables entre sí (Cuadro 1): 1) medición de la proteinuria en una muestra de orina tomada al azar; 2) medición de la proteinuria en una orina de 24 hrs, con medición simultánea del aclaramiento de creatinina.

La literatura mundial aconseja otros métodos adicionándoles: 1) la orina minutada y 2) la relación albúmina/creatinina. No obstante han recibido mejor aceptación los mencionados anteriormente, por su confiabilidad y su fácil realización, sobre todo el tomar una sola muestra de orina por la mañana. Además, la literatura internacional ha resaltado la equivalencia en los resultados de estos dos métodos principalmente en el manejo de la macroalbuminuria.

Las mediciones de la proteinuria tienen variaciones individuales, por lo que se deben recolectar 2 valores anormales dentro de un período de 3-6 meses para afirmar su diagnóstico. ${ }^{11,12,14}$

\begin{tabular}{|ccc|}
\hline \multicolumn{3}{|c|}{$\begin{array}{c}\text { Cuadro 1 } \\
\text { Los diferentes valores de proteinuria }\end{array}$} \\
\hline Categoría & $\begin{array}{c}\text { Orina de 24 hrs } \\
\text { (mg/24h) }\end{array}$ & $\begin{array}{c}\text { Muestra de orina } \\
\text { al azar }\end{array}$ \\
\hline $\begin{array}{c}\text { Normal } \\
\text { Microalbuminuria } \\
\text { (nefropatía incipiente) } \\
\text { Macroalbuminuria } \\
\text { (nefropatía abierta) }\end{array}$ & $30-300$ & $<30$ \\
\hline * Determinado por el Diastik. & $>300$ & $>300$ \\
\hline
\end{tabular}

\begin{tabular}{|ccc|}
\hline \multicolumn{3}{|c|}{$\begin{array}{c}\text { Cuadro 2 } \\
\text { Detección de Microalbuminuria por el Micral test II }\end{array}$} \\
\hline Color & $\begin{array}{c}\text { Letra impresa } \\
\text { en el rótulo }\end{array}$ & Rangos mg/l \\
\hline A & Negativo & $0-15$ \\
B & $20 \mathrm{mg} / \mathrm{l}$ & $8-35$ \\
C & $50 \mathrm{mg} / \mathrm{l}$ & $30-80$ \\
D & $100 \mathrm{mg} / \mathrm{l}$ & $70-260$ \\
\hline
\end{tabular}

Existen condiciones que pueden producir microalbuminuria transitoria: 1) hiperglicemia de corto tiempo, no controlada; 2) ejercicio intenso; 3) infección de vías urinarias, 4) hipertensión arterial no controlada, 5) insuficiencia cardíaca congestiva y 6) enfermedades febriles agudas.

La evaluación de la proteinuria se puede realizar en forma anual inicialmente por medio de la tirilla, (el test Dipstick o Diastik), que detecta la albuminuria > de $300 \mathrm{mg} /$ día. Este método se ha comparado con la orina de 24 horas y tiene una sensibilidad del $100 \%$ y una especificidad del $95,7 \%$. Además, es fácil de realizar en el consultorio y su costo es bajo. ${ }^{12-15}$

Si el Dipstick es positivo y se han descartado los factores que puedan alterar el resultado como anteriormente se expuso, se puede llegar al diagnóstico de nefropatía abierta. Si el Dipstick es negativo, hay que determinar la presencia de microalbuminuria.

La microalbuminuria se puede realizar por la tirilla "Micral test II". Hay varios estudios en los que se ha demostrado su sensibilidad y especificidad, comparándola con la recolección de orina de $24 \mathrm{~h}$. Este método se basa en una gama de colores (A-D) que representan diferentes valores ${ }^{16}$ (Cuadro 2).

Para el punto de $20 \mathrm{mg} / \mathrm{l}$ hay una sensibilidad del $96,7 \%$ y una especificidad del $71 \%$. El valor predictivo negativo fue de 0,95 y el positivo de 0,78 , con un $52 \%$ de prevalencia de positividad en las muestras. ${ }^{16}$ 
Junto con la detección de microalbuminuria con el "Micral test II" se debe así mismo descartar las posibles alteraciones transitorias y repetir el método cada 3 ó 6 meses. Si es positivo en 2 ocasiones distintas, ello hace el diagnóstico de microalbuminuria y se debe iniciar el tratamiento.

Todas estas pruebas cualitativas se deben confirmar con métodos específicos. El seguimiento de estos pacientes debe de ser mínimo 2 veces al año, con el propósito de vigilar la respuesta al tratamiento.

\section{Fisiopatología y factores de riesgo}

Para el desarrollo de la ND probablemente se requiera la presencia de varios factores.

Hiperglucemia: La evidencia que respalda el papel de la hiperglucemia en la patogénesis de la ND, deriva del estudio del Diabetic Control and Complications Trial (DCCT), ${ }^{17}$ que demostró cómo la terapia intensificada con insulina $\left(\mathrm{HbA}_{1} \mathrm{c}<7 \%\right)$ disminuyó en un $50 \%$ el desarrollo de la ND.

Estudios más recientes, como el United Kingdom Prospective Diabetes Study (UKPDS) en DM tipo 2, ${ }^{12,13}$ evidencian una disminución del 29\% de la microalbuminuria y del 39\% de riesgo para la proteinuria.

Otras ventajas del control glucémico se han evidenciado en el Stockholm Interventión Study y el estudio de Kumamoto. ${ }^{11}$

Fisiopatológicamente, la hiperglucemia puede inducir en forma directa la expansión mesangial, ya sea incrementando la producción de la matriz mesangial, o por la glicosilación de las proteínas de la misma o de las proteínas tisulares.

Existe una glicosilación inicial, que es una unión no enzimática covalente y luego se producen las formas avanzadas e irreversibles de glicosilación o productos finales, por medio de un efecto de rearreglo "Amadori"; estos últimos tienden a unirse al colágeno contribuyendo al desarrollo de la ND.

La hiperglucemia puede alterar la permeabilidad capilar, permitiendo el paso de proteína mediante la cápsula de Bowman, lo cual produce lesiones tóxicas tisulares, tanto por ellas mismas, como por los ácidos grasos que por su alta afinidad con la albúmina, viajan junto a ella.

La acumulación de lípidos en las células tubulares próximales es responsable de la hipoxia tisular. Toda esta injuria inicial provoca cambios inflamatorios que llevan finalmente a la fibrosis y cicatrización del túbulo e intersticio.

Como consecuencia de la acumulación de proteínas en cantidades excesivas en el fluído del túbulo proximal, se estimula la producción de endotelinas en el compartimento intersticial renal, en donde ejercen funciones inflamatorias y vaso activas. Estas moléculas tienen receptores en los fibroblastos, que además de la activación del factor de crecimiento Beta, llevan a la producción del componente de la matriz extracelular por el fibroblasto, especialmente por el colágeno tipo IV, laminina y fibronectina; al mismo tiempo se eleva la producción de citokinas mediadoras de la inflamación a partir de estas células y de las mismas células del epitelio tubular, contribuyendo a la fibrosis.

Hipertensión arterial: En los DM tipo 1 la hipertensión arterial generalmente es evidente al tiempo del desarrollo de la microalbuminuria, y en los DM tipo 2 puede estar presente al momento del diagnóstico. La hipertensión sistólica o distólica acelera la progresión de la ND y el agresivo tratamiento antihipertensivo es capaz de disminuir el promedio de la caída de la FG.

En el UKPDS, ${ }^{18-21}$ se documentó una reducción significativa en todos los puntos finales relacionados con la DM en un $24 \%$, un $32 \%$ de las muertes relacionadas con DM y un $37 \%$ de las complicaciones microvasculares (95\% IC 11-56\% $\mathrm{p}=0,0092),{ }^{20,21}$ en normotensión.

En la fisiopatología de la ND puede contribuir la hiperfiltración glomerular en donde la proteinuria juega un papel importante, aunada a la hiperglucemia.

La hipótesis de la hiperfiltración glomerular se ha tomado como uno de los factores de progresión de la enfermedad renal; pero en estudios recientes no se ha visto una relación lineal entre estos factores, pues aún pacientes sin hiperfiltración progresan hacia el deterioro renal, y pacientes con hiperfiltración no necesariamente progresan hacia la nefropatía abierta mientras otros factores, como el pobre control metabólico, ${ }^{22,23}$ juegan un papel más importante en dicho deterioro.

Existe también la asociación entre historia familiar de hipertensión arterial (HTA) y el desarrollo subsecuente de hipertensión y proteinuria.

La hipertensión glomerular puede ocurrir independientemente de la HTA sistémica, y probablemente resulte en la disminución de las resistencias vasculares intrarrenales. En estudios experimentales, se asocia la vasodilatación de la arteriola aferente y vasoconstricción de la arteriola eferente en etapas iniciales, lo cual produce estrés con hipertensión glomerular. Desde hace tres décadas H. Keen, dijo que: "existen dos malas compañías para la diabetes: hipertensión e hiperglucemia", ambas asociadas con la microalbuminuria. El UKPDS ${ }^{18-21}$ extendió estas malas compañías, incluyendo la dislipidemia y el fumado.

En un estudio de los DM tipo $2^{19,20,24}$ se pudo documentar que existen tres factores de riesgo importantes para el desarrollo de la ND estos son: presión arterial media > de 95mmhg; $\mathrm{HbA}_{1 \mathrm{c}}>$ del $9 \%$ y colesterol > de $203 \mathrm{mg} / \mathrm{dl}$; donde su relación de riesgo fue de 43 (95\% IC 25-106 p < 0,01); el riesgo para el progreso hacia la microalbuminuria durante el seguimiento fue de 18 (95\% IC 11-33, p=0,01) en relación con los de poco riesgo. Escasos niveles de HDL-colesterol, el cigarrillo y bajo estrato socioeconómico en los varones, estuvo asociado con el desarrollo de la ND. ${ }^{24}$ 
Enfermedad cardiovascular: La microalbuminuria es un indicador temprano de la progresión de la enfermedad renal y la mortalidad cardiovascular en DM..$^{3-5}$ La presencia de microalbuminuria también incrementa 15 veces más el riesgo de mortalidad cardiovascular. ${ }^{3,13,25}$ La macroalbuminuria se ha asociado con un aumento del riesgo de mortalidad cardiovascular, 37 veces más que la población general y 4,7 veces más que en diabéticos con normoalbuminuria. ${ }^{10}$

En DM tipo 2, la microalbuminuria predice la alta tasa de mortalidad por enfermedad arterial coronaria. Pacientes DM tipo 1 con nefropatía abierta desarrollan extensa enfermedad aterosclerótica. Todo paciente con DM tipo 1 ó $2 \geq 35$ años de edad con la presencia de microalbuminuria persistente o nefropatía abierta, amerita un adecuado examen cardiológico. ${ }^{25}$

Sin embargo, la microalbuminuria no es un factor de riesgo independiente para todas las causas de morbimortalidad por enfermedad coronaria. En DM tipo 2 es atribuible a los efectos de otros factores de riesgo cardiovascular ${ }^{5}$ como: sexo masculino, enfermedad coronaria previa, $\mathrm{Hb}_{i} \mathrm{c}>8 \%$, hipercolesterolemia y fumado.

Entre los varones libres de enfermedad coronaria de base, los factores de riesgos independientes de la morbi-mortalidad por ésta fueron: el fumado, hipertensión diastólica e hipercolesterolemia.

La microalbuminuria precede a la enfermedad coronaria, aunque no se puede excluir la posibilidad que esta pudiera ya estar presente en forma subclínica en dichos pacientes. En DM tipo 1, la microalbuminuria se ha asociado con cardiopatía isquémica silente.

Dado que la microalbuminuria precede el desarrollo de nueva enfermedad coronaria y la persistencia de esta precede el desarrollo de microalbuminuria en el varón diabético tipo 2 , se piensa que puede ser el resultado de un determinante común de injuria vascular.

La hipótesis que relaciona la enfermedad coronaria y microalbuminuria, incluye: "insulinoresistencia", aumento de la permeabilidad vascular, disfunción endotelial y alteraciones en la aterogenicidad de las partículas lipoproteicas ante la presencia de hiperglucemia.

Retinopatía: Se ha relacionado la ND con el grado de retinopatía diabética, de tal manera que cuando se evidencia nefropatía sin retinopatía, es poco probable que sea de etiología diabética. ${ }^{6}$

Se ha reportado que en los diabéticos tipo 2, la prevalencia de retinopatía varía de $47 \%$ a $63 \%$ en pacientes con proteinuria, ${ }^{6}$ sugiriendo esto que cerca del $30 \%$ de ellos tienen proteinuria de etiología no diabética, en donde la asociación más común es la glomerulopatía membranosa, en la cual el $12 \%$ de los pacientes presentó retinopatía.

Fumado: Es un fuerte predictor de la ND, siendo de tres a cuatro veces más común el desarrollo de ND en los pacientes que fuman, que en los que no lo hacen, presentando un riesgo intermedio aquellos pacientes que dejan de fumar.

Dislipidemia: Se ha asociado la presencia de dislipidemia con el riesgo de progresión de nefropatía incipiente a nefropatía abierta. La elevación del colesterol y triglicéridos en algunos estudios tienen un riesgo relativo de $1,4(1,1-1,7)$ $\mathrm{p}<0,05 .{ }^{26}$ En un estudio con estatinas (Simvastatina) se mostró una disminución de un $25 \%$ de la EUA en pacientes normotensos-microalbuminúricos. ${ }^{9}$

Susceptibilidad genética: En ambos tipos de DM hay evidencias que sugieren la susceptibilidad genética, la cual aumenta la posibilidad de desarrollo de ND, en pacientes cuyos hermanos o padres la hayan presentando.

En los indígenas Pima la proteinuria ocurrió en un $14 \%$ de los descendientes sin ningún pariente con ND en un $23 \%$ si uno de los padres tenía ND, y en un $46 \%$ si ambos padres la tuvieron. ${ }^{12,13}$

El gen de la enzima convertidora de angiotensina puede contribuir a la susceptibilidad genética y al polimorfismo, inserción/delección de dicho gen, puede modular la respuesta de la progresión de ND en los DM tipo 1, como se evidenció en el EURODIAB, ${ }^{7,8}$ La distribución genotípica fue del $15 \%$ II, $58 \%$ ID y el $27 \%$ DD; encontrando que el tipo II tuvo mayor progresión de la excreción urinaria de albúmina y fue el grupo que tuvo más respuesta al Lisinopril.

Dieta: En cuanto a la dieta, el efecto de la restricción de proteínas a un valor aproximado de $0.7 \mathrm{~g} / \mathrm{Kg} / \mathrm{d}$ áa, ha tenido un efecto modesto sobre el retardo de la caída de la FG. Pudiera restringirse a $0,8 \mathrm{~g} / \mathrm{Kg} / \mathrm{día}$, aportando así el $10 \%$ de las calorías diarias en pacientes con nefropatía abierta. ${ }^{11,13}$

En vista de todo lo antes expuesto, se desprenden acciones básicas en cuanto al manejo de los pacientes:

- Estricto control glucémico ya documentado en el DCCT, el UKPDS. En este último se presentó igual disminución del riesgo de complicaciones microvasculares utilizando insulina, sulfonilureas o metformina. ${ }^{17-19}$

- Control de la HTA, tratando de alcanzar una tensión sistólica menor de $130 \mathrm{mmHg}$ y diastólica menor de 85 $\mathrm{mm} \mathrm{Hg}$, y en los hipertensos sistólicos > de $180 \mathrm{mmHg}$, inicialmente reducir su tensión arterial a $<$ de $160 \mathrm{~mm}$ $\mathrm{Hg}$ y buscar una disminución en $20 \mathrm{~mm} \mathrm{Hg}$ si la tensión sistólica está entre 160-179 mm Hg. ${ }^{11}$ Se comprobaron iguales efectos benéficos sobre las complicaciones micro y macrovasculares con el uso del captopril y atenolol. ${ }^{21}$ El efecto en microalbuminúricos y normoalbuminúricos obedece básicamente a las disminuciones aún ligeras en la presión arterial.

Luego de esta revisión podría concluirse que: a) a todo paciente DM tipo 1, luego de 5 años de haber sido diagnosticado, y a todo paciente DM tipo 2 al momento del diagnóstico, se le debe realizar la medición de proteinuria. b) Las tiras 
reactivas para determinar la micro y macroalbuminuria son confiables en relación con la orina de $24 \mathrm{~h}$. c) Los factores más importantes dentro de la evolución de la nefropatía diabética y que exigen un agresivo manejo son: un buen control metabólico y el manejo antihipertensivo.

\section{Abstract}

Background: Due to the growing incidence of Diabetes Mellitus in a younger population, and in view of the poor level of metabolic control reached worldwide for this illness, it is necessary to find simple and practical methods, within our reach, in order to detect the appearance of the chronic complications of Diabetes in very early stages to avoid or at least slow down the natural evolution of them.

Aim: To review the most recent evidence of the utility and readiness of different methods to evaluate albuminuria, in order to improve the approach of the diabetic patient, with or without nephropathy.

Methodology: Bibliographical review of publications of the last 10 years.

Conclusions: a) All patient with DM type 1, 5 years after their diagnosis and all diabetic patient type 2 at the moment of the diagnosis should have a measurement of their proteinuria. b) The reactivate strips to determine the micro and macro-albuminuria are reliable and correlate well with the meansurement of albuminuria in a 24 hours orine. c) The most important factors that inside in the evolution of the diabetic nephropathy and that demand an aggressive management are: a good metabolic control and the control of the blood presure.

\section{Referencias}

1. Winocour Ph. Microalbuminuria. BMJ 1992, 304: 1196-7.

2. Jarret RJ, Viberti GC, Argyropulus A, Hill RD, Mahmud U, Murrells TJ. Microalbuminuria predicts mortality in non- Insulin-Dependent Diabetes. Diabetes Med 1984,1:17-19.

3. Mogensen CE. Microalbuminuria Predicts Clinical Proteinuria and Early Mortality in Maturity Onset Diabetes. N Engl J Med 1984; 310: 35660 .

4. Viverti GC, Hill RD, Jarret RJ, Argyropoulus A, Mahmud U, Keen H. Microalbuminuria as a predictor of clinical Nephropathy in InsulinDependent Diabetes. Lancet 1982; i1430-32.

5. Mattock MB, Barnes DJ, Viberti JC, Keen H, Burt D, Hughes JM, Fitzgerald AP. Microalbuminuria and Coronary Heart Disease in NIDDM. Diabetes 1998; 47: 1786-92.

6. Nauchin MK, Kawano T, Uyama H, Shaiiki H, Dohi K. Discordance between retinopathy and nefropathy in type 2 Diabetes. Nephron 1998; 80:171-74.

7. Pennoi G, Chaturved N, Tolmud J PhD, Cotroneo P, Manto A, Nannipieri M, Loung L, Fuller JH and the EUCLID Study Group. Diabetes 1998; 47: 1507-11.

8. The EUCLID Study Group. Randomized Placebo-Controlled trial of Lisinopril in Normotensive Patients with Insulin-dependent Diabetes and Normoalbuminuria or Microalbuminuria. Lancet 1997; 349:1787-92.
9. Tonolo G, Ciccaresse M, Brizzi P, Puddu L, Zecchi G y cols. Reduction of Albumin Excretion Rate in Normotensive Microalbuminuric type 2 Diabetic Patients During Long-Term Simvastatin Treatment. Diabetes Care. 1997; 20: 1891-95.

10. Bakris GL, Stein JH. Diabetes Nephropathy. Disease-a-month 1993; 39: $573-612$

11. Diabetic Nephropathy Position Statement, American Diabetes Association. Diabetes Care 1999; 22 (1): A66-A69.

12. Morrison G, Neilson EG, Sheeram A, Alan G, Wassertein SM, Kobonin SM. Diabetic Nephropathy. Disease a Month 1998; 44 (5): 214-34.

13. Poinier Zuny SJ. New México: Preserving the Diabetic kidney. The Am J of Family Practice 1998; 46: 21-7.

14. Bennet PH, Haffner S. Kasike BL, Keane WF, Mongensen CE, Parving H. Diabetic Renal Disease Recomendations: Am J of Kidney Diseases $1995 ; 45: 107-112$

15. Zelmanovitz T, Gross JL, Oliveira J, de Acevedo MJ. Proteinuria is Still Useful For The Screenning and Diagnosis of Overt Diabetic Nephrophaty. Diabetes Care 1998; 21: 1076-79.

16. Mogensen C, Viberti GC, Petrein E, Kuter D, Hasslacher Ch, Hoffman W, et al. Multicenter Evaluation of the Micral-Test II. Test Strip and Inmunologic Rapid Test for The Detection of Microalbuminuria. Diabetes Care 1997; 20: 1642-46.

17. The Diabetes Control and Complications Trial Research Group. The effect of Intensive Treatment of Diabetes on the Development and progession of long-term complications in insulino-dependent Diabetes. $\mathrm{N}$ Engl J Med 1993; 329:977-86.

18. UK Prospective Diabetes Study Group. Intensive Blood Glucose Control with Sulphonylureas or Insulin Compared with conventional treatment and risk of complication in patients with type 2 Diabetes (UKPDS 33). Lancet $1998 ; 352: 837-53$.

19. UK Prospective Diabetes Study Group. Effect of intensive Blood Glucose control policy with metformin on complication in Type 2 Diabetes (UKPDS 34). Lancet 1998; 352:854-65.

20. UK Prospective Diabetes Study Group. Tight Blood Presure Control and Risk of Macrovascular and Microvascular complication in Type 2 Diabetes (UKPDS 38). BMJ 1998; 317: 703-12.

21. UK Prospective Diabetes Study Group. Efficacy of Atenolol and Captopril in Reducing. Risk of Macrovascular and Microvascular complication in Type 2 Diabetes (UKPDS 39). BMJ 1998; 317:713-25.

22. Bangstad H, Osterby R, Hartmann A, Berg TJ, Hanssen KF. Severity of Glomerulopathy predicts Long-Term Urinary Albumin Excretión Rate in Patients With Type 1 Diabetes and Microalbuminuria. Diabetes Care 1999; 22: 314-19.

23. Chaiken RL, Eckert-Norton M, Bard M, Banerji M, Palmisano J, Sachimechi I, Levovitz HE. Hiperfiltration in African-American patients with type 2 Diabetes. Diabetes Care 1998; 21: 2129-34.

24. Ranid M, Brosh D, Ravid-Safran D, Levi Z, Rachmani Z. Main Risk Factores For Nephropathy in type 2 Diabetes Mellitus are plasma Cholesterol levels; mean Blood Presure and hyperglycemia. Arch Intern Med 1998; 158: 998-1004.

25. American Diabetes Association. Consensus Development Conference on the Diagnosis of Coronary Heart Disease in People with Diabetes. Diabetes Care 1998; 21: 1551-55.

26. Annegall M, Hougaard PhD, Borch- Johnsen K, Parving H. Risk Factor for Development of incipient and overt Diabetes Nephropathy in Patients with non-Insulin Dependent Diabetes Mellitus, Prospective Observational Study. BMJ 1997, 314: 783-88. 